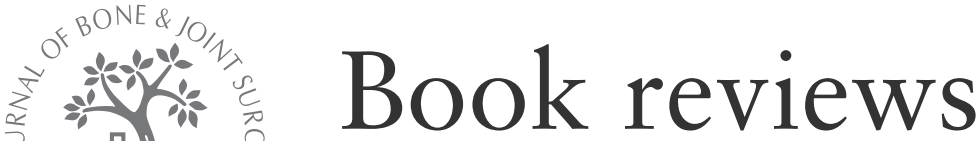

(C)British Editorial Society of Bone and Joint Surgery

doi:10.1302/0301-620X.90B9.21435 \$2.00 J Bone Joint Surg [Br] 2008;90-B:1260.

\section{Beyond Thalidomide: Birth defects explained.}

\section{Edited by J. McCredie}

Pp. 418. Royal Society of Medicine Press Ltd. 2007. ISBN: 13: 978-1-85315741-7. £35.

It was a pleasure to read this outstanding and scholarly monograph, the fruit of Professor Janet McCredie's 30 years' experience in the field. The book puts together beautifully, through clear prosem excellent illustrations and an extensitve bibliography, the story of thalidomide and the spin-offs there from.

In the description of the thalidomide epidemic between 1958 and 1961, we are reminded that the malformations were throughout the body; virtually no organ was spared. Thalidomide had the ability to exaggerate naturally occurring malformations and Professor McCredie's driving force over the succeeding years was the hypothesis that if the mode of action of thalidomide could be established, the pathogenesis of other sporadic malformations might also be revealed. Several chapters take us through the pharmacology of thalidomide, its use by tetratologists to induce malformations in laboratory animals and its ability to cause neuropathies. There is also an extensive clinical and radiological investigation of the resultant skeletal malformations.

Two chapters remind us of the embryonic neural crest, the precursor to sensory and autonomic nerves, and we are introduced to Professor McCredie's next hypothesis, that the underlying mechanism of congenital malformations is an injury to the neural crest. She presents experimental and pathological evidence to support this hypothesis, and a rational approach to congenital defect syndromes. The hypothesis rests on the acceptance that the normal development of a limb, for example, depends on the trophic influence of the embryonic sensory neurone. We are also shown how neural crest injury might cause defects in other structures such as those which meet in the mid-line, and then affecting cylindrical or solid organs. We then move to neurotropism, whereby growth and repair are dependent upon nerves, especially sensory, and that neuritrophism is quantitative, in that sufficient nerves must be present for growth or regeneration to occur. With regard to limb deficiencies, we are shown that limb buds have a natrual nerve supply which is diminished in limb deficient rabbits who had been fed thalidomide.

We are then given an explanation of sclerotomes as skeletal elements supplied by one spinal segmental nerve. Examples of sclerotome subtraction are shown logically to be related to the various limb reductions seen clinically. Other chapters explain multiple malformations and those affecting internal organs. We are left with the firm opinion that the teratogenic effect of thalidomide is due to its neurotoxicity and that further knowledge on malformations is likely to be gained through research into neurotoxicology.

I am virtually convinced by Professor McCredie's arguments. However, I can think of some examples in clinical practice which might raise doubt. In the conditions of spina bifida, sacral agenesis or lumbo-sacral agenesis, where there is extensive loss or absence of sensory nerves, why do we not get more associated reduction malformations? But I congratulate Professor McCredie on her book and hope it achieves the landmark status it certainly deserves.

D. Jones

\section{Decision making in spinal care}

Edited by D. G. Anderson, A. R. Vaccaro

Pp. 504. Thieme: New York, 2007. ISBN: 12:978-3-13-140481-7. Euro 60.

This succinct volume covers virtually the whole field of spinal surgery but it is very orientated towards operative treatments or, as noted in the preface, "a focused tool" for clinicians. Treatment algorthms are included and plenty of figures and tables pack the text with helpful data.

A substantial proportion of the book deals with spinal injuries and, although non-operative treatment options are addressed, there is little guidance on the management of spinal cord injury or the specialist assessment of the paraplegic patient; nor for that matter is the issue of prioritisation of the treatment of any associated injury addressed.

The suggested readings with short summaries in the references at the end of each chapter is an attractive feature and includes key historical papers. The section on tuberculosis of the spine is somewhat disappointing in view of the fact that this condition has become more common in recent years with the rise of HIV/Aids. Posterior infection is not mentioned nor is the very topical issue of drug resistance.

Spinal monitoring and its role is well described and is indeed a welcome chapter. New technologies such as disc nucleus replacement, vertibroplasty, computer assisted surgery and non-fusion techniques are all discussed. The text retains a sense of proportion when stressing that the evidence of beneficial outcome is weak for some of these developments. The comment that traditional approaches to the cervical spine already fulfil many of the goals set for less invasive surgical approaches is welcome.

The book seeks to inform over a very broad spectrum of spinal conditions providing an introduction to further study and will be a very valuable guide for surgical trainees and established consultants in trauma and orthopaedics. The specialist spinal surgeon will also find help with less common problems and I commend it. Most texts on spinal conditions are huge but this book is a convenient size. I am sure we shall see a new edition before long.

A. G. Thompson 